\title{
Distribution of human papillomavirus types, cervical cancer screening history, and risk factors for infection in Manitoba
}

\author{
A. A. Demers, PhD (1,2,3); B. Shearer, PhD (4); A. Severini, MD (5); R. Lotocki, MD, FRCSC (6,7); \\ E. V. Kliewer, PhD (2,3,8); S. Stopera, MSc (9); T. Wong, MD, FRCPC $(1,10) ;$ G. Jayaraman, PhD $(1,11)$
}

This article has been peer reviewed.

\begin{abstract}
Objectives: We conducted a study to investigate the prevalence of human papillomavirus (HPV) infections in an opportunistic sample of women in Manitoba, Canada. We inquired about risk factors associated with HPV infections and linked the HPV typing results with the cervical cancer screening history of the participants.
\end{abstract}

Methods: The study population included 592 women attending Papanicolaou (Pap) test clinics. After signing a consent form, participants were given a self-administered questionnaire on risk factors and received a conventional Pap test. Residual cells from the Pap tests were collected and sent for HPV typing.

Results: The mean age of the population was 43 years. A total of 115 participants $(19.4 \%)$ had an HPV infection, 89 of whom had a normal Pap test. Of those who were HPV-positive, 61 (10.3\%) had high-risk (Group 1) HPV. HPV-16 was the most prevalent type (15/115: 13.0\% of infections). The most consistent risk factors for HPV infection were young age, Aboriginal ethnicity, higher lifetime number of sexual partners and higher number of sexual partners in the previous year.

Conclusion: The prevalence of HPV types in Manitoba is consistent with the distributions reported in other jurisdictions. These data provide baseline information on type-specific HPV prevalence in an unvaccinated population and can be useful in evaluating the effectiveness of the HPV immunization program. An added benefit is in the validation of a proof of concept which links a population-based Pap registry to laboratory test results and a risk behaviour survey to assess early and late outcomes of HPV infection. This methodology could be applied to other jurisdictions across Canada where such capacities exist.

Keywords: papillomavirus infections, prevalence, risk factors, uterine cervical dysplasia, early detection of cancer

\section{Introduction}

The publicly funded human papillomavirus (HPV) immunization programs implemented across Canada between 2007 and 2009 have the potential to prevent a large proportion of anogenital warts, high-grade cervical lesions and HPV-related invasive cancers. ${ }^{1-6}$ They also have the potential to influence cervical cancer screening as currently practiced because of the changes in prevalence of cervical abnormalities they can bring about., ${ }^{1,7}$ The extent of this impact, however, will depend on the distribution of HPV types, the type-specific infection rates among females and the vaccine uptake.

The objective of this study was to determine the baseline type-specific prevalence of and risk factor for HPV infection in an opportunistic sample of women attending walk-in, no-appointment Papanicolaou (Pap) test clinics in Manitoba (Canada) during an annual cervical cancer awareness week. The survey information and HPV typing results were linked to the Manitoba Cervical Cancer Screening Program (MCCSP) database. Manitoba is well positioned to host and conduct this kind of surveillance projects because of the availability of linkable population-based databases on cancer,

Author references:

1. Centre for Communicable Diseases and Infection Control, Public Health Agency of Canada, Ottawa, Ontario, Canada

2. Department of Epidemiology and Cancer Registry, CancerCare Manitoba, Winnipeg, Manitoba, Canada

3. Department of Community Health Sciences, University of Manitoba, Winnipeg, Manitoba, Canada

4. International Centre for Infectious Diseases, Winnipeg, Manitoba, Canada

5. National Microbiology Laboratory, Public Health Agency of Canada, Winnipeg, Manitoba, Canada

6. Gynecological Oncology, CancerCare Manitoba, Winnipeg, Manitoba, Canada

7. Manitoba Cervical Cancer Screening Program, Manitoba, Canada

8. Cancer Control Research, British Columbia Cancer Agency, Vancouver, British Columbia, Canada

9. Communicable Disease Control Branch, Public Health and Primary Health Care, Manitoba Health, Winnipeg, Manitoba, Canada

10. Department of Infectious Diseases, University of Ottawa, Ottawa, Ontario, Canada

11. Department of Epidemiology and Community Medicine, University of Ottawa, Ottawa, Ontario, Canada

Correspondence: Alain Demers, Centre for Communicable Diseases and Infection Control, Public Health Agency of Canada, 100 Eglantine Driveway, Tunney's Pasture, AL 0602C, Ottawa, ON K1A 0K9; Tel.: (613) 948-8247; Fax: (613) 941-9813; Email: alain.demers@phac-aspc.gc.ca 
cervical screening, medical procedures provided by physicians and immunization. ${ }^{8}$ These resources provide a robust environment to evaluate the impact of the HPV immunization program impact, the utilization of cervical screening among vaccinated and non-vaccinated females, and the resulting disease distribution and outcomes.

\section{Methods}

\section{Study environment}

Since 2003 the MCCSP has conducted an annual Pap Week in October. During this week women are encouraged to attend Pap test clinics without appointment. The objective is to reach those who have never attended or do not regularly attend cervical screening. In 2008, 123 clinics participated in Pap Week across Manitoba. Of these, 52 consented to take part in this study. In addition to performing conventional Pap tests, these clinics took the residual cells from the Pap tests, put them in a liquid-based cytology medium, and sent the samples to Cadham Provincial Laboratory in Winnipeg, Manitoba, for HPV typing. The participating clinics also supervised the administration of a consent form and a self-administered survey on risk factors for HPV infections.

\section{Population}

The study population was composed of an opportunistic sample of women aged 18 years and older from different ethnic backgrounds. Pregnant women were excluded. Women interested in participating in the study discussed the objectives with clinic staff and, upon agreement, signed a consent form and completed a risk factor questionnaire. Women who decided not to complete the questionnaire were still eligible for HPV testing, and their HPV results were included in the analysis.

The study was publicized on posters in the clinics, and staff told potential participants about it. Overall, 1182 women underwent cervical screening in the 52 participating clinics, and 642 (54\%) consented to participate in the study.

\section{Follow-up of participants}

Health care providers received the Pap test results and the HPV typing results. Medical management of participants diagnosed with cervical abnormalities followed the MCCSP cervical cancer screening management guidelines in effect at the time of the study. Women who tested positive for high-risk HPV and negative for cytology were recalled by the clinics for further investigation according to the MCCSP guidelines.

\section{Risk factor survey}

The survey included questions on sociodemographic characteristics and relevant risk factors for cervical neoplasia including smoking, oral contraceptive use, recent sexual activity, previous diagnosis with sexually transmitted infections and HPV immunization status. The questionnaire was tested to a grade four reading level before use.

\section{Cervical specimen processing and $H P V$ detection and typing}

The Luminex assay is a method developed at the National Microbiology Laboratory that detects 45 HPV types. These include 23 of the 25 high-risk (as defined by the International Agency for Research on Cancer) types found in groups $1,2 \mathrm{a}$ and 2b: HPV types 16, 18, 26, 30, 31, 33, 35, $39,45,51,52,53,56,58,59,66,67,68$, 69, 70, 73, 82 and $85 .{ }^{9}$ Also included are 22 types considered of low risk or unknown risk: HPV types 6, 11, 13, 32, $40,42,43,44,54,61,62,71,72,74,81$, $83,84,86,87,89,90$ and 91. In brief, samples in viral transport medium were centrifuged and their DNA extracted from the resulting pellet using a MagnaZorb DNA extraction kit. $^{10,11}$ The DNA was amplified with a nested polymerase chain reaction (PCR) method using the general PGMY primer set for the first round ${ }^{12}$ and the GP5+/GP6+ primer set for the second. ${ }^{13}$ This method amplifies a fragment of the L1 region of the HPV genome (about 150 base pairs in length). The quality of the DNA sample for PCR was checked by co-amplification of the human beta-globin gene. PCR products were visually detected by gel electrophoresis. ${ }^{13-16}$

HPV DNA was detected and typed by hybridization to microspheres coupled to specific probes for the $45 \mathrm{HPV}$ types according to the xMAP Luminex technology*. Specificity and sensitivity of this method for all the 45 types of HPV was measured using cloned HPV DNAs. Comparison against the LinearArray (Roche) ${ }^{17}$ and other HPV genotyping kits showed that this Luminex assay is comparable to other commercial genotyping methods. $^{18}$

\section{Data analysis}

HPV typing results and survey results were linked to the MCCSP database using a unique identifier in order to get the results of the Pap tests performed during Pap Week 2008 and the cervical cancer screening history of the consenting participants. Univariate and multivariate logistic regression analyses was used to calculate odds ratios (ORs) and 95\% confidence intervals (CIs) as estimates of the relative risk of HPV detection associated with the various predictor variables. Because of the higher prevalence of HPV in women aged less than 30 years, results were tabulated for women aged less than 30 years and for those aged 30 years plus. HPV types were grouped according to Bouvard et al. and de Villiers et al. ${ }^{9,19}$

The protocol was approved by the research ethics boards of Health Canada/ Public Health Agency of Canada and the University of Manitoba.

\section{Results}

Tissue samples collected from the 642 women who consented to participate in the study were sent for HPV infection testing. Of these, 33 women did not complete the consent form and were excluded from the analyses. A further 17 were excluded because of inadequate samples. The final study population included 592 participants, of which 527 completed the questionnaire. The 
mean age of the study population was 43 years (median: 44). The mean age of infected women was 35 years (median: 31 years), and the mean age of noninfected women was 45 years (median: 46 years). The majority of participants came from rural areas (66.3\%), and the remainder came from Winnipeg and Brandon.

\section{Survey results}

Variables associated with the HPV infection using univariate analysis are reported in Table 1. Results are presented for women aged less than 30 years (referred to as "younger") and for women aged 30 years and older (referred to as "older") to reflect the higher prevalence of HPV infections in younger women. In older women, HPV infection was associated with Aboriginal ethnicity and a self-described difficult financial situation. Compared with non-smokers, participants who smoked were at greater risk of being HPV-positive, regardless of age. Not having a history of

\section{TABLE 1}

Survey results by age and HPV infection status

\begin{tabular}{|c|c|c|c|c|c|c|c|c|c|c|c|}
\hline \multirow[t]{3}{*}{ Variables $^{\mathrm{a}}$} & \multirow[t]{3}{*}{ Categories } & \multicolumn{5}{|c|}{ Age $<30$ years } & \multicolumn{5}{|c|}{ Age $\geq 30$ years } \\
\hline & & \multicolumn{2}{|c|}{ HPV- $(n=75)$} & \multicolumn{2}{|c|}{$\mathrm{HPV}+(\mathrm{n}=56)$} & \multirow[t]{2}{*}{ OR $(95 \% \mathrm{CI})$} & \multicolumn{2}{|c|}{ HPV- $(n=402)$} & \multicolumn{2}{|c|}{ HPV $+(n=59)$} & \multirow[t]{2}{*}{ OR $(95 \% \mathrm{Cl})$} \\
\hline & & $\mathbf{n}$ & $\%$ & $\mathbf{n}$ & $\%$ & & $\mathbf{n}$ & $\%$ & $\mathbf{n}$ & $\%$ & \\
\hline \multirow{3}{*}{ Ethnic identity } & Caucasian & 38 & $(50.7)$ & 24 & $(42.9)$ & Reference & 276 & $(68.7)$ & 26 & $(44.1)$ & Reference \\
\hline & Other & 10 & (13.3) & 3 & (5.4) & $0.5 \quad(0.1,1.9)$ & 26 & (6.5) & 5 & (8.5) & $2.0 \quad(0.7,5.8)$ \\
\hline & Not stated & 9 & $(12.0)$ & 10 & (17.9) & $1.8 \quad(0.6,5.0)$ & 42 & (10.4) & 10 & (16.9) & $2.5 \quad(1.1,5.6)$ \\
\hline \multirow{4}{*}{ Financial situation } & Moderate & 20 & (26.7) & 14 & $(25.0)$ & $0.9 \quad(0.4,2.1)$ & 110 & (27.4) & 18 & $(30.5)$ & $1.7 \quad(0.9,3.4)$ \\
\hline & Comfortable & 32 & (42.7) & 25 & $(44.6)$ & Reference & 201 & $(50.0)$ & 19 & $(32.2)$ & Reference \\
\hline & Very comfortable & 9 & (12.0) & 2 & (3.6) & $0.3 \quad(0.1,1.4)$ & 32 & (8.0) & 5 & (8.5) & $1.7 \quad(0.6,4.7)$ \\
\hline & Not stated & 9 & (12.0) & 10 & (17.9) & $1.4(0.5,4.0)$ & 40 & (10.0) & 11 & (18.6) & $2.9 \quad(1.3,6.5)$ \\
\hline Education & High school or less & 28 & (37.3) & 19 & (33.9) & Reference & 139 & (34.6) & 18 & $(30.5)$ & Reference \\
\hline \multirow{3}{*}{ Currently smoking } & Former smoker & 7 & (9.3) & 6 & (10.7) & $2.0 \quad(0.6,6.7)$ & 103 & (25.6) & 11 & (18.6) & $1.1 \quad(0.5,2.6)$ \\
\hline & Never & 39 & (52.0) & 17 & (30.4) & Reference & 159 & (39.6) & 15 & $(25.4)$ & Reference \\
\hline & Not stated & 8 & (10.7) & 10 & (17.9) & $2.9(1.0,8.5)$ & 39 & (9.7) & 9 & (15.3) & $2.5 \quad(1.0,6.0)$ \\
\hline \multirow{4}{*}{$\begin{array}{l}\text { Currently use oral } \\
\text { contraceptive }\end{array}$} & Yes & 24 & $(32.0)$ & 15 & $(26.8)$ & $0.7 \quad(0.3,1.6)$ & 18 & (4.5) & 3 & (5.1) & $1.3 \quad(0.4,4.6)$ \\
\hline & No & 31 & $(41.3)$ & 27 & $(48.2)$ & Reference & 292 & (72.6) & 38 & $(64.4)$ & Reference \\
\hline & Don’t know & 2 & (2.7) & 1 & (1.8) & - & 1 & $(0.2)$ & 1 & (1.7) & - \\
\hline & Not stated & 18 & $(24.0)$ & 13 & $(23.2)$ & $0.8 \quad(0.3,2.0)$ & 91 & (22.6) & 17 & (28.8) & $1.4 \quad(0.8,2.7)$ \\
\hline \multirow[t]{3}{*}{ Ever had a Pap test } & Yes & 47 & $(62.7)$ & 41 & $(73.2)$ & Reference & 357 & (88.8) & 46 & (78.0) & Reference \\
\hline & No & 20 & (26.7) & 5 & (8.9) & $0.3(0.1,0.8)$ & 7 & (1.7) & 2 & (3.4) & $2.2(0.4,11.0)$ \\
\hline & Don’t know & 0 & $(0.0)$ & 0 & $(0.0)$ & - & 0 & $(0.0)$ & 2 & (3.4) & - \\
\hline \multirow{4}{*}{ Number of children } & 1 & 10 & (13.3) & 9 & $(16.1)$ & $1.4 \quad(0.5,3.8)$ & 33 & (8.2) & 10 & (16.9) & $1.5 \quad(0.5,3.9)$ \\
\hline & 2 & 7 & (9.3) & 5 & (8.9) & $1.1 \quad(0.3,3.7)$ & 113 & (28.1) & 9 & (15.3) & $0.4 \quad(0.2,1.0)$ \\
\hline & $\geq 3$ & 4 & (5.3) & 2 & (3.6) & $0.8 \quad(0.1,4.4)$ & 167 & $(41.5)$ & 21 & (35.6) & $0.6 \quad(0.3,1.4)$ \\
\hline & Not stated & 10 & (13.3) & 11 & (19.6) & $1.7 \quad(0.6,4.4)$ & 41 & $(10.2)$ & 9 & (15.3) & $1.1 \quad(0.4,2.8)$ \\
\hline \multirow{5}{*}{$\begin{array}{l}\text { Number of sexual partners } \\
\text { over last year }\end{array}$} & 0 & 7 & (9.3) & 2 & (3.6) & $0.7 \quad(0.1,3.8)$ & 74 & (18.4) & 11 & (18.6) & $1.4 \quad(0.7,2.9)$ \\
\hline & $>0^{\mathrm{b}}$ & 0 & $(0.0)$ & 1 & (1.8) & - & 7 & (1.7) & 1 & (1.7) & - \\
\hline & 1 & 48 & $(64.0)$ & 19 & (33.9) & Reference & 280 & $(69.7)$ & 30 & $(50.8)$ & Reference \\
\hline & 2 or more & 15 & $(20.0)$ & 26 & $(46.4)$ & $4.4(1.9,10.0)$ & 12 & (3.0) & 11 & (18.6) & $8.6(3.5,21.1)$ \\
\hline & Not stated & 5 & (6.7) & 8 & (14.3) & $4.0(1.2,13.9)$ & 29 & (7.2) & 6 & (10.2) & $1.9 \quad(0.7,5.0)$ \\
\hline
\end{tabular}


TABLE 1 (continued)

Survey results by age and HPV infection status

\begin{tabular}{|c|c|c|c|c|c|c|c|c|c|c|c|}
\hline \multirow[t]{3}{*}{ Variables $^{\mathrm{a}}$} & \multirow[t]{3}{*}{ Categories } & \multicolumn{5}{|c|}{ Age $<30$ years } & \multicolumn{5}{|c|}{ Age $\geq 30$ years } \\
\hline & & \multicolumn{2}{|c|}{ HPV- $(n=75)$} & \multicolumn{2}{|c|}{ HPV+ $(n=56)$} & \multirow[t]{2}{*}{ OR $(95 \% \mathrm{CI})$} & \multicolumn{2}{|c|}{ HPV $-(n=402)$} & \multicolumn{2}{|c|}{ HPV+ $(n=59)$} & \multirow[t]{2}{*}{ OR $(95 \% \mathrm{Cl})$} \\
\hline & & $\mathbf{n}$ & $\%$ & n & $\%$ & & $\mathbf{n}$ & $\%$ & n & $\%$ & \\
\hline \multirow{5}{*}{$\begin{array}{l}\text { Lifetime number } \\
\text { of sexual partners }\end{array}$} & 0 & 6 & $(8.0)$ & 2 & (3.6) & $1.0 \quad(0.2,5.6)$ & 12 & $(3.0)$ & 3 & $(5.1)$ & $3.3(0.9,13.0)$ \\
\hline & $>0^{\mathrm{b}}$ & 5 & $(6.7)$ & 1 & (1.8) & - & 23 & (5.7) & 4 & $(6.8)$ & - \\
\hline & $1-4$ & 36 & $(48.0)$ & 12 & $(21.4)$ & Reference & 227 & $(56.5)$ & 17 & (28.8) & Reference \\
\hline & $\geq 5$ & 24 & $(32.0)$ & 33 & (58.9) & $4.1 \quad(1.8,9.5)$ & 111 & $(27.6)$ & 28 & $(47.5)$ & $3.4(1.8,6.4)$ \\
\hline & Not stated & 4 & (5.3) & 8 & (14.3) & $6.0(1.5,23.5)$ & 29 & (7.2) & 7 & (11.9) & $3.2(1.2,8.4)$ \\
\hline \multirow{4}{*}{$\begin{array}{l}\text { Had unprotected anal sex } \\
\text { over last year }\end{array}$} & Yes & 14 & (18.7) & 10 & (17.9) & $0.9 \quad(0.4,2.4)$ & 38 & $(9.5)$ & 7 & (11.9) & $0.8 \quad(0.3,1.8)$ \\
\hline & No & 52 & $(69.3)$ & 35 & $(62.5)$ & Reference & 307 & (76.4) & 43 & (72.9) & Reference \\
\hline & Don't know & 0 & $(0.0)$ & 1 & (1.8) & - & 3 & $(0.7)$ & 0 & $(0.0)$ & - \\
\hline & Not stated & 9 & $(12.0)$ & 10 & (17.9) & $1.6 \quad(0.5,5.2)$ & 54 & $(13.4)$ & 9 & $(15.3)$ & $0.9 \quad(0.3,2.6)$ \\
\hline \multirow{4}{*}{$\begin{array}{l}\text { Currently in a stable } \\
\text { relationship }\end{array}$} & Yes & 54 & $(72.0)$ & 23 & $(41.1)$ & Reference & 289 & (71.9) & 35 & $(59.3)$ & Reference \\
\hline & No & 11 & $(14.7)$ & 19 & (33.9) & $4.1 \quad(1.7,9.9)$ & 64 & (15.9) & 12 & $(20.3)$ & $1.5(0.8,3.1)$ \\
\hline & Not sure & 1 & $(1.3)$ & 4 & $(7.1)$ & - & 4 & $(1.0)$ & 3 & $(5.1)$ & - \\
\hline & Not stated & 9 & $(12.0)$ & 10 & (17.9) & $2.6 \quad(0.9,7.3)$ & 45 & $(11.2)$ & 9 & (15.3) & $1.7 \quad(0.7,3.7)$ \\
\hline \multirow[t]{3}{*}{ Total number of Pap tests ${ }^{c}$} & 0 & 22 & (29.3) & 14 & $(25.0)$ & $0.8 \quad(0.3,1.8)$ & 43 & $(10.7)$ & 11 & (18.6) & $2.1(1.0,4.5)$ \\
\hline & $1-4$ & 30 & $(40.0)$ & 25 & (44.6) & Reference & 270 & $(67.2)$ & 33 & (55.9) & Reference \\
\hline & $5+$ & 23 & (30.7) & 17 & $(30.4)$ & $0.9 \quad(0.4,2.0)$ & 89 & $(22.1)$ & 15 & $(25.4)$ & $1.4(0.7,2.7)$ \\
\hline \multirow[t]{2}{*}{ Total number of colposcopies ${ }^{c}$} & 0 & 64 & $(85.3)$ & 49 & (87.5) & Reference & 379 & $(94.3)$ & 55 & $(93.2)$ & Reference \\
\hline & $1+$ & 11 & $(14.7)$ & 7 & (12.5) & $0.8 \quad(0.3,2.3)$ & 23 & $(5.7)$ & 4 & $(6.8)$ & $1.2 \quad(0.4,3.6)$ \\
\hline \multirow[t]{3}{*}{ Worst cytologyc } & No history & 22 & $(29.3)$ & 14 & $(25.0)$ & $1.0 \quad(0.5,2.4)$ & 43 & $(10.7)$ & 11 & (18.6) & $2.0(1.0,4.3)$ \\
\hline & Normal & 42 & $(56.0)$ & 26 & $(46.4)$ & Reference & 323 & (80.3) & 40 & $(67.8)$ & Reference \\
\hline & Other than normal & 11 & $(14.7)$ & 16 & (28.6) & $2.4 \quad(0.9,5.8)$ & 36 & $(9.0)$ & 8 & (13.6) & $1.8 \quad(0.8,4.1)$ \\
\hline \multirow[t]{3}{*}{ Worst histologyc } & No history & 64 & $(85.3)$ & 49 & (87.5) & Reference & 379 & (94.3) & 55 & $(93.2)$ & Reference \\
\hline & Normal & 3 & $(4.0)$ & 2 & (3.6) & $0.9 \quad(0.1,5.4)$ & 12 & $(3.0)$ & 1 & $(1.7)$ & $0.6 \quad(0.1,4.5)$ \\
\hline & Other than normal & 8 & (10.7) & 5 & (8.9) & $0.8 \quad(0.3,2.7)$ & 11 & $(2.7)$ & 3 & (5.1) & $1.9 \quad(0.5,6.9)$ \\
\hline
\end{tabular}

Abbreviations: ASC-H, atypical squamous cells-cannot rule out high-grade lesion; ASC-US, atypical squamous cells of unknown significance; $\mathrm{Cl}$, confidence interval; CIN, cervical intraepithelial neoplasia; HPV, human papillomavirus; HPV-, HPV-negative; HPV+, HPV-positive; HSIL, high-grade squamous intraepithelial lesions; LSIL, low-grade squamous intraepithelial lesions; OR, odds ratio; Pap, Papanicolaou; STI, sexually transmitted infection.

Note: Bolded values are significant.

${ }^{a}$ Variables are all self-reported.

${ }^{b}$ Value obtained by combining information on the number of children and sexual activity questions.

c Manitoba Cervical Cancer Screening Program data; other cytology: ASC-US, LSIL, ASC-H, HSIL; other histology: CIN I, CIN II, CIN III; all the other variables are self-reported by the participants.

sexually transmitted infections (STIs) was protective for HPV infection for both age groups. Women with a higher number of lifetime sexual partners or a higher number of sexual partners over the previous year were more likely to be HPV-positive. Younger women who were not in a stable relationship were more likely to be HPV-positive than those in a stable relationship or older women.

In the multivariate logistic regression analysis, being younger (OR $=0.97$; 95\% CI: 0.95-0.99; age was treated as a continuous variable), Aboriginal $(\mathrm{OR}=4.83$; $95 \%$ CI: 2.70-8.65; compared to non-Aboriginal), and having two or more sexual partners in the previous year
(OR $=2.04 ; 95 \%$ CI: $1.20-3.47$ compared to one or no sexual partner) were significant predictors for testing HPV-positive. The variables that were not significant predictors of HPV infection in the multivariate model were currently smoking (yes/no), Pap test history (yes/no), history of cervical abnormality (yes/no) and having had at least two consecutive screening events within a year (yes/no).

\section{Reported and registry-based Pap test history}

Older women who had had zero Pap tests between 2001 (the year the MCCSP database was started) and October 2008 were at higher risk of being HPV-positive (Table 1; data from the MCCSP). A similar trend was observed with Pap test history, although the number of respondents who had had no Pap test was small. Younger women who self-reported not ever having a Pap test were at lower risk of having an HPV infection, although this was not observed when the analyses were performed with the MCCSP data.

\section{HPV infections and cytological outcomes}

A total of 115 participants (19\%) were found to be HPV-positive (Table 2). Overall, $33 \%(38 / 115)$ of these infections were among participants aged less than 25 years. The participants aged less than 25 years were also more likely to be infected with Group 1 HPV types 
TABLE 2

Age distribution of women by infection status and HPV type (person-based)

\begin{tabular}{|c|c|c|c|c|c|c|c|c|c|c|c|}
\hline \multirow[t]{2}{*}{$\begin{array}{l}\text { Age, } \\
\text { years }\end{array}$} & HPV- & $\mathrm{HPV}^{\mathrm{a}}$ & \multicolumn{2}{|c|}{ Group $1^{b}$} & $\begin{array}{c}\text { HPV } \\
16 \text { or } 18^{c}\end{array}$ & Group $2^{d}$ & \multicolumn{2}{|c|}{$\begin{array}{c}\text { HPV } \\
6 \text { or } 11^{c}\end{array}$} & Low-risk ${ }^{e}$ & $\begin{array}{c}\text { Multiple } \\
\text { infections }\end{array}$ & Total \\
\hline & n $\quad(\%)$ & n $\quad(\%)$ & $\mathbf{n}$ & (\%) & n $\quad(\%)$ & n $\quad(\%)$ & n & $(\%)$ & n $\quad(\%)$ & n $\quad(\%)$ & (\%) \\
\hline$<25$ & $40 \quad(8.4)$ & $38(33.0)$ & 27 & (44.3) & $8(40.0)$ & $4(21.1)$ & & (75.0) & $13(29.5)$ & $15(50.0)$ & $78(13.2)$ \\
\hline $25-29$ & $35 \quad$ (7.3) & 18 (15.7) & 9 & (14.8) & $4(20.0)$ & $3(15.8)$ & & (25.0) & 7 (15.9) & $4(13.3)$ & $53(9.0)$ \\
\hline $30-34$ & $46 \quad(9.6)$ & $5 \quad(4.3)$ & 5 & $(8.2)$ & $2(10.0)$ & $\begin{array}{ll}0 & (0.0)\end{array}$ & 0 & $(0.0)$ & $0 \quad(0.0)$ & 1 & $51 \quad(8.6)$ \\
\hline $35-39$ & $41 \quad(8.6)$ & $9 \quad(7.8)$ & 2 & (3.3) & $\begin{array}{ll}0 & (0.0)\end{array}$ & 3 (15.8) & 0 & $(0.0)$ & $4 \quad(9.1)$ & 1 (3.3) & 50 \\
\hline $40-44$ & $66(13.8)$ & 11 (9.6) & 6 & (9.8) & $1 \quad(5.0)$ & 1 & 0 & $(0.0)$ & $5(11.4)$ & $2(6.7)$ & $77(13.0)$ \\
\hline $45-49$ & 56 (11.7) & $14(12.2)$ & 4 & (6.6) & $1 \quad(5.0)$ & $3(15.8)$ & 0 & $(0.0)$ & 7 (15.9) & $3(10.0)$ & $70(11.8)$ \\
\hline 50-54 & $62(13.0)$ & 10 (8.7) & 5 & $(8.2)$ & $2(10.0)$ & $2(10.5)$ & 0 & $(0.0)$ & $4 \quad(9.1)$ & $3(10.0)$ & $72(12.2)$ \\
\hline 55-59 & $51(10.7)$ & $5 \quad(4.3)$ & 2 & (3.3) & $2(10.0)$ & $1 \quad(5.3)$ & 0 & $(0.0)$ & 2 & $\begin{array}{ll}0 & (0.0)\end{array}$ & $56 \quad(9.5)$ \\
\hline $60-64$ & $36 \quad(7.5)$ & $4 \quad(3.5)$ & 1 & (1.6) & $\begin{array}{ll}0 & (0.0)\end{array}$ & $2(10.5)$ & 0 & $(0.0)$ & 1 & 1 (3.3) & 40 \\
\hline $65+$ & $44 \quad(9.2)$ & $1 \quad(0.9)$ & 0 & $(0.0)$ & $\begin{array}{ll}0 & (0.0)\end{array}$ & $\begin{array}{ll}0 & (0.0)\end{array}$ & 0 & $(0.0)$ & 1 & $\begin{array}{ll}0 & (0.0)\end{array}$ & $45 \quad(7.6)$ \\
\hline Total & 477 & 115 & 61 & & 20 & 19 & 4 & & 44 & 30 & 592 \\
\hline
\end{tabular}

Abbreviation: HPV, human papillomavirus.

${ }^{\text {a }}$ Any HPV type included in Group 1, Group 2, and low-risk (see text); note that HPV 34 and 97, which belong to Group $2,{ }^{9}$ are not included in the HPV types covered by the methodology used in this study.

b Group 1: HPV 16, 18, 31, 33, 35, 39, 45, 51, 52, 56, 58, 59.

c Either one type or both present at the same time.

${ }^{d}$ Group 2: HPV 26, 30, 53, 66, 67, 68, 69, 70, 73, 82, 85.

e Low-risk: HPV 6, 11, 13, 32, 40, 42, 43, 44, 54, 61, 62, 71, 72, 74, 81, 83, 84, 86, 87, 89, 90, 91.

(44\%; 27/61) than Group 2 types $(21 \%$; 4/19). While HPV types 6 and 11 were not detected in women aged 30 years plus, HPV types 16 and 18 (but mostly 16) were detected over a wider age range. One-quarter of the infected women $(26 \% ; 30 / 115)$ had multiple HPV infections, that is, more than one HPV of any type.

Of the study population with a normal Pap test, $17 \%$ (89/517) tested positive for an HPV infection and 9\% (46/517) were infected with Group 1 HPV (Table 3). Overall, 7\% (41/592) of all participants had an abnormal Pap test result. An HPV infection (any type) was found in $11 \%$ of unsatisfactory Pap tests $(2 / 18), 32 \%$ of atypical squamous cells of unknown significance (ASC-US; 6/19), 63\% of low-grade squamous intraepithelial lesions (LSIL; 10/16) and $75 \%$ of high-grade squamous intraepithelial lesions (HSIL; $3 / 4)$. Group $1 \mathrm{HPV}$ type was found in $6 \%$ of unsatisfactory Pap tests (1/18), $11 \%$ of ASC-US (2/19), 38\% of LSIL (6/16) and $75 \%$ of HSIL $(3 / 4)$. Group $1 \mathrm{HPV}$ types (overall: $10.3 \%$ [61/592]; among HPVinfected participants: $53.0 \%$ [61/115]) were detected more frequently than Group 2 (overall: $3.2 \%$ [19/592]; among HPV-infected participants: 16.5\% [19/115]) and low-risk HPV types (overall: $7.4 \%$ [44/592]; among HPV-infected participants: $38.2 \%[44 / 115])$. Pap test results were not available for $3 \%(16 / 592)$ of the HPV samples tested.
It is not clear why some Pap tests were not sent to the lab for evaluation. We suspect that the clinicians that performed these tests understood that taking a tissue sample for HPV typing

TABLE 3

Person-based HPV prevalence by cytological outcome

\begin{tabular}{|c|c|c|c|c|c|c|c|c|c|}
\hline \multirow{2}{*}{$\begin{array}{l}\text { HPV } \\
\text { types }\end{array}$} & \multirow{2}{*}{$\begin{array}{c}\text { Missing } \\
\text { n }\end{array}$} & \multirow{2}{*}{$\begin{array}{c}\text { Normal } \\
n\end{array}$} & \multirow{2}{*}{$\begin{array}{c}\text { Unsatis- } \\
\text { factory } \\
\text { n }\end{array}$} & \multirow{2}{*}{$\begin{array}{c}\text { ASC-US } \\
\mathbf{n}\end{array}$} & \multirow{2}{*}{$\begin{array}{l}\text { LSIL } \\
\text { n }\end{array}$} & \multirow{2}{*}{$\begin{array}{c}\text { ASC-H } \\
\mathbf{n}\end{array}$} & \multirow{2}{*}{$\begin{array}{c}\text { HSIL } \\
n\end{array}$} & \multicolumn{2}{|c|}{ Total } \\
\hline & & & & & & & & $\mathbf{n}$ & $\%$ \\
\hline Negative & 11 & 428 & 16 & 13 & 6 & 2 & 1 & 477 & 80.6 \\
\hline Any $^{\mathrm{a}}$ & 5 & 89 & 2 & 6 & 10 & 0 & 3 & 115 & 19.4 \\
\hline 6 or $11^{b}$ & 0 & 3 & 1 & 0 & 0 & 0 & 0 & 4 & 0.7 \\
\hline 16 & 2 & 10 & 0 & 1 & 2 & 0 & 0 & 15 & 2.5 \\
\hline 16 or $18^{b}$ & 2 & 14 & 0 & 1 & 2 & 0 & 1 & 20 & 3.4 \\
\hline Group $1^{c}$ & 3 & 46 & 1 & 2 & 6 & 0 & 3 & 61 & 10.3 \\
\hline Group $2^{\mathrm{d}}$ & 1 & 13 & 0 & 2 & 2 & 0 & 1 & 19 & 3.2 \\
\hline Low-risk $^{\mathrm{e}}$ & 1 & 36 & 1 & 3 & 3 & 0 & 0 & 44 & 7.4 \\
\hline Multiple $^{f}$ & 1 & 22 & 0 & 2 & 4 & 0 & 1 & 30 & 5.1 \\
\hline Total & 16 & 517 & 18 & 19 & 16 & 2 & 4 & 592 & \\
\hline
\end{tabular}

Abbreviations: ASC-H, atypical squamous cells-cannot rule out high-grade lesion;

ASC-US, atypical squamous cells of unknown significance; HPV, human papillomavirus;

HSIL, high-grade squamous intraepithelial lesions; LSIL, low-grade squamous intraepithelial lesions.

${ }^{\text {a }}$ Any HPV type included in Group 1, Group 2, and low-risk (see following text); note that HPV 34 and 97, which belong to Group 2, ${ }^{9}$ are not included in the HPV types covered by the methodology that was used in this study.

${ }^{b}$ One type or the other or both can be present at the same time.

${ }^{c}$ Group 1: HPV 16, 18, 31, 33, 35, 39, 45, 51, 52, 56, 58, 59.

${ }^{d}$ Group 2: HPV 26, 30, 53, 66, 67, 68, 69, 70, 73, 82, 85.

e Low-risk: HPV 6, 11, 13, 32, 40, 42, 43, 44, 54, 61, 62, 71, 72, 74, 81, 83, 84, 86, 87, 89, 90, 91.

${ }^{\mathrm{f}}$ Multiple HPV infections. 
was their only task for this study and did not request a regular cytological testing.

Among Group 1 types, HPV-16 (10\%) was the most frequently detected followed by HPV-39 (5\%), 58 (5\%), 18 (4\%), 35 (4\%), $51(4 \%), 52(4 \%), 59(4 \%)$ and $33(3 \%)$ (Table 4). Within the genus alpha, species 9 (29\%; 45/157), 3 (19\%; 29/157) and 7 $(17 \% ; 26 / 157)$ were the most frequently detected. Species 9 includes viruses related to HPV-16, while species 7 includes those related to HPV-18, and species 3 includes low-risk HPV types.

\section{Discussion}

Comparing the prevalence of HPV infections across studies is difficult because typing technologies, sampled populations and sampling strategies are often different. In addition, prevalence rates are rarely age-standardized. With this in mind, a meta-analysis reported HPV infection rates as varying from $7 \%$ to $8 \%$ in Europe and Asia, $14 \%$ in North America, and $23 \%$ in Africa in women with normal cytology. ${ }^{20}$ In the United States, rates have been estimated as $27 \%$ in females aged 14 to 59 years. ${ }^{21}$ Our study found an HPV prevalence of $19 \%$ (17\% among those with a normal Pap test). HPV-16 was the most prevalent cervical type detected, while other common high-risk types included types $18,33,35,39,51,52,58$ and 59. These results are consistent with other findings where HPV types 16, 18, 31, 39, $51,52,56$ and 58 were found to be among the most frequent types worldwide in women with normal cytological findings; ${ }^{22}$ HPV types 16, 18, 31, 33, 45, 51, 52, 56 and 58 in women diagnosed with low-grade cervical lesions; ${ }^{23}$ and HPV types 16, 18, 31, $33,35,45,52$ and 58 in women diagnosed with high-grade abnormalities. ${ }^{24}$ HPV type-specific prevalence rankings, however, varied regionally and by country. ${ }^{22-24}$ For example, a Belgium population-based study reported that the most common high-risk type was HPV-16 (3.7\%), followed by types 31, 51 and 53, which were identified in at least $2 \%$ of the population (HPV-18 was found in $1.5 \%$ of the population). ${ }^{25} \mathrm{~A}$ Swedish population-based study reported infection prevalence for HPV-16 of $2.5 \%$, followed by HPV-31 (1.4\%), HPV-45 (0.9\%)
TABLE 4

Infection-based prevalence of the HPV genital species of the alpha genus

\begin{tabular}{|c|c|c|c|c|c|c|c|c|c|}
\hline & \multirow{2}{*}{$\begin{array}{l}\text { HPV } \\
\text { types }\end{array}$} & \multirow{2}{*}{$\begin{array}{c}\text { Missing } \\
\text { n }\end{array}$} & \multirow{2}{*}{$\begin{array}{c}\text { Negative } \\
\text { n }\end{array}$} & \multirow{2}{*}{$\begin{array}{c}\text { Unsatis- } \\
\text { factory } \\
\mathbf{n}\end{array}$} & \multirow{2}{*}{$\begin{array}{c}\text { ASC-US } \\
\mathbf{n}\end{array}$} & \multirow{2}{*}{$\begin{array}{l}\text { LSIL } \\
\text { n }\end{array}$} & \multirow{2}{*}{$\begin{array}{c}\text { HSIL } \\
n\end{array}$} & \multicolumn{2}{|c|}{ Total } \\
\hline & & & & & & & & n & $\%$ \\
\hline \multirow[t]{3}{*}{ A1 } & 32 & 0 & 4 & 0 & 0 & 0 & 0 & 4 & 2.5 \\
\hline & 42 & 0 & 6 & 0 & 0 & 0 & 0 & 6 & 3.8 \\
\hline & Total & 0 & 10 & 0 & 0 & 0 & 0 & 10 & 6.4 \\
\hline \multirow[t]{8}{*}{ A3 } & 62 & 0 & 5 & 0 & 1 & 0 & 0 & 6 & 3.8 \\
\hline & 72 & 0 & 2 & 0 & 0 & 0 & 0 & 2 & 1.3 \\
\hline & 81 & 0 & 5 & 0 & 0 & 1 & 0 & 6 & 3.8 \\
\hline & 83 & 0 & 3 & 0 & 0 & 1 & 0 & 4 & 2.5 \\
\hline & 84 & 0 & 2 & 0 & 0 & 0 & 0 & 2 & 1.3 \\
\hline & 86 & 0 & 2 & 0 & 0 & 0 & 0 & 2 & 1.3 \\
\hline & 89 & 0 & 6 & 0 & 0 & 1 & 0 & 7 & 4.5 \\
\hline & Total & 0 & 25 & 0 & 1 & 3 & 0 & 29 & 18.5 \\
\hline \multirow[t]{4}{*}{ A5 } & 51 & 0 & 4 & 0 & 0 & 1 & 1 & 6 & 3.8 \\
\hline & 69 & 0 & 1 & 0 & 0 & 0 & 0 & 1 & 0.6 \\
\hline & 82 & 0 & 1 & 0 & 0 & 0 & 0 & 1 & 0.6 \\
\hline & Total & 0 & 6 & 0 & 0 & 1 & 1 & 8 & 5.1 \\
\hline \multirow[t]{5}{*}{ A6 } & 30 & 0 & 2 & 0 & 0 & 1 & 0 & 3 & 1.9 \\
\hline & 53 & 0 & 0 & 0 & 0 & 1 & 1 & 2 & 1.3 \\
\hline & 56 & 0 & 3 & 0 & 0 & 0 & 0 & 3 & 1.9 \\
\hline & 66 & 0 & 4 & 0 & 0 & 0 & 0 & 4 & 2.5 \\
\hline & Total & 0 & 9 & 0 & 0 & 2 & 1 & 12 & 7.6 \\
\hline \multirow[t]{7}{*}{ A7 } & 18 & 0 & 5 & 0 & 0 & 0 & 1 & 6 & 3.8 \\
\hline & 39 & 1 & 4 & 0 & 0 & 1 & 1 & 7 & 4.5 \\
\hline & 45 & 0 & 1 & 0 & 0 & 0 & 0 & 1 & 0.6 \\
\hline & 59 & 0 & 5 & 0 & 0 & 1 & 0 & 6 & 3.8 \\
\hline & 70 & 0 & 4 & 0 & 1 & 0 & 0 & 5 & 3.2 \\
\hline & 85 & 0 & 1 & 0 & 0 & 0 & 0 & 1 & 0.6 \\
\hline & Total & 1 & 20 & 0 & 1 & 2 & 2 & 26 & 16.6 \\
\hline \multirow[t]{4}{*}{ A8 } & 7 & 0 & 1 & 0 & 0 & 0 & 0 & 1 & 0.6 \\
\hline & 40 & 0 & 1 & 0 & 2 & 0 & 0 & 3 & 1.9 \\
\hline & 91 & 0 & 0 & 0 & 1 & 0 & 0 & 1 & 0.6 \\
\hline & Total & 0 & 2 & 0 & 3 & 0 & 0 & 5 & 3.2 \\
\hline \multirow[t]{8}{*}{ A9 } & 16 & 2 & 10 & 0 & 1 & 2 & 0 & 15 & 9.6 \\
\hline & 31 & 0 & 2 & 0 & 1 & 0 & 0 & 3 & 1.9 \\
\hline & 33 & 0 & 5 & 0 & 0 & 0 & 0 & 5 & 3.2 \\
\hline & 35 & 0 & 4 & 0 & 0 & 1 & 1 & 6 & 3.8 \\
\hline & 52 & 0 & 6 & 0 & 0 & 0 & 0 & 6 & 3.8 \\
\hline & 58 & 1 & 4 & 1 & 0 & 1 & 0 & 7 & 4.5 \\
\hline & 67 & 1 & 1 & 0 & 0 & 1 & 0 & 3 & 1.9 \\
\hline & Total & 4 & 32 & 1 & 2 & 5 & 1 & 45 & 28.7 \\
\hline \multirow[t]{5}{*}{ A10 } & 6 & 0 & 2 & 0 & 0 & 0 & 0 & 2 & 1.3 \\
\hline & 11 & 0 & 1 & 1 & 0 & 0 & 0 & 2 & 1.3 \\
\hline & 44 & 1 & 3 & 0 & 1 & 0 & 0 & 5 & 3.2 \\
\hline & 74 & 0 & 5 & 0 & 0 & 0 & 0 & 5 & 3.2 \\
\hline & Total & 1 & 11 & 1 & 1 & 0 & 0 & 14 & 8.9 \\
\hline A11 & 73 & 0 & 0 & 0 & 1 & 0 & 0 & 1 & 0.6 \\
\hline A13 & 54 & 0 & 4 & 0 & 0 & 1 & 0 & 5 & 3.2 \\
\hline \multirow[t]{3}{*}{ Other } & 8 & 0 & 1 & 0 & 0 & 0 & 0 & 1 & 0.6 \\
\hline & 38 & 0 & 0 & 0 & 0 & 1 & 0 & 1 & 0.6 \\
\hline & Total & 0 & 1 & 0 & 0 & 1 & 0 & 2 & 1.3 \\
\hline Total & & 6 & 120 & 2 & 9 & 15 & 5 & & \\
\hline
\end{tabular}

Abbreviations: ASC-H, atypical squamous cells-cannot rule out high-grade lesion;

ASC-US, atypical squamous cells of unknown significance; HPV, human papillomavirus;

HSIL, high-grade squamous intraepithelial lesions; LSIL, low-grade squamous intraepithelial lesions. 
and HPV-18 (0.7\%); $13.0 \%$ of women had multiple infections. ${ }^{26}$ Many studies have reported an increase in HPV infections in women 60 years of age and older. ${ }^{27}$ There were insufficient cases to confirm that trend in Manitoba.

A few studies have investigated the prevalence of HPV in Canada. A British Columbia study found an overall HPV prevalence rate of $16.8 \%$ (high-risk HPV: 13.9\%; HPV-16: 10.7\%);28 an Ontario study found an overall infection rate of $13.3 \%$ (high-risk HPV: 9.6\%, HPV-16: 7.3\%); ${ }^{29} \mathrm{~A}$ New Brunswick study found a prevalence of 28\% (high-risk HPV: $21 \%$ ). ${ }^{30}$ A study conducted between 1992 and 1995 recruited a large proportion of Aboriginal women $(42 \%)$ from a clinic located in a low-income inner-city area of Winnipeg, Manitoba, and found that HPV infections rates in Aboriginal and non-Aboriginal women were comparable $(33.6 \%$ and $31.8 \%$, respectively). ${ }^{31}$ However, because of the different populations included in our present study and this earlier one, comparison of results is difficult.

The prevalence of high-risk HPV has been consistently reported to increase with the severity of lesions. For example, a meta-analysis reported high-risk HPV in $71.9 \%$ (95\% CI: $62.8 \%-80.9 \%$ ) of LSIL cases $^{23}$ and $88.3 \% \quad(95 \%$ CI: $85.8 \%-$ $90.8 \%$ ) of HSIL cases. ${ }^{24}$ Moore et al. ${ }^{28}$ reported that $52.3 \%$ of LSIL and $79.4 \%$ of HSIL contained high-risk HPV. They also found that HPV positivity increased from normal $(12.3 \%)$ to benign $(19.6 \%)$ to low-grade $(69.3 \%)$ to high-grade $(81.0 \%) .^{28}$ We found $37.5 \%$ of LSIL were high-risk (Group 1) HPV-positive, as were $75 \%$ of HSIL.

A number of cofactors are associated with risk of having an HPV infection and different grades of cervical abnormalities, many of which are related to sexual behaviours. The factors that have been the most consistently associated with higher rates of HPV infections include younger age and having a greater number of lifetime and recent sex partners. ${ }^{32,33}$ Other cofactors for HPV infection, including age at sexual debut, smoking, oral contraceptive use, ethnicity, alcohol consumption, history of STI, income, and condom use have also been reported, but not consistently. ${ }^{33-41}$ The multivariate analysis showed that age, ethnicity, and the number of sexual partners in the last year were independent predictors. Our present study also suggests that some of these risk factors are common for all age groups while other factors are found only in either younger or older women.

Women with no history of cervical cancer screening and those who were under-screened have been reported to have higher incidence rates of cervical cancer than women who regularly received screening. ${ }^{42-45}$ In the present study, women 30 years of age and older with no Pap test history were found to be HPV-positive more often.

\section{Limitations of the study}

The present study has several limitations. As with almost all seroprevalence studies, our study relied on opportunistic samples and was not population-based. Consequently, the results do not necessarily represent the rate of HPV infections in the general female population. The publicity made around Pap Week in Manitoba and the clinics dedicated to one-day screening could also create a selection bias by encouraging symptomatic women who have delayed screening to finally get a Pap test. It is difficult to predict the outcome of such bias on the current risk factor analysis, but if it is differential, it may explain why the risk of infection was higher in some groups of people. The cervical screening participation rate in Manitoba between 2007 and 2009 in women aged 20 to 69 years was $65.9 \%$. The breakdown of their cytological results was normal cytology, 95.5\%; ASC-US $3.1 \%$; LSIL $2.1 \%$; atypical glandular cells (AGC) $0.1 \%$; ASC-H $0.3 \%$; and HSIL $0.9 \%$. Among study participants, the cervical screening participation rate since 2001 was $84.8 \%$ (502/592), with a breakdown of cytology results of normal cytology $87 \%$ (517/592); ASC-US $3 \%(19 / 592)$; LSIL $3 \%$ $(16 / 592) ; \quad$ ASC-H $0.3 \% \quad(2 / 592) ;$ and HSIL 1\% (4/592).

This comparison suggests that most of the study participants attend cervical screening regularly and that their cytological outcomes were comparable to the women who attended cervical screening in Manitoba between 2007 and 2009. The cross-sectional nature of the study design does not allow for establishing a causal relationship between HPV infection and the cofactors investigated. In addition, self-administered questionnaires can be subject to biases. Nevertheless, findings are consistent with current knowledge on risk factors for HPV infections. Due to the high sensitivity of the HPV detection method, the clinical significance of the present study is limited. The PCR amplification can detect as little as one copy of the targeted genes (L1 DNA), and this sensitivity does not necessarily translate into infection of clinical significance. Depuydt et al. showed that below a critical viral load, detection of visually detectable lesions is very rare. ${ }^{46}$ A highly sensitive test has the potential to limit the triaging of people with HPV infections.

\section{Conclusion}

The results from our study suggest that the distribution of oncogenic HPV types in Manitoba is in accordance with what has been reported in Canada and in other countries. These data provide a baseline of HPV prevalence in an unvaccinated population in Manitoba. In addition, the use of data linkage provides a proof of concept for the applicability of population-based registry linkage to evaluate HPV immunization programs in those jurisdictions where the capacity to conduct such linkages exist.

\section{References}

1. Garnett GP, Kim JJ, French K, Goldie SJ. Chapter 21: Modelling the impact of HPV vaccines on cervical cancer and screening programmes. Vaccine. 2006;24 Suppl 3:S178-86.

2. Brisson M, Van de Velde N, Boily M-C. Different population-level vaccination effectiveness for HPV types 16, 18, 6 and 11. Sex Transm Infect. 2011;87(1):41-3.

3. Smith MA, Canfell K, Brotherton JML, Lew J-B, Barnabas RV. The predicted impact of vaccination on human papillomavirus infections in Australia. Int $\mathrm{J}$ Cancer. 2008;123(8):1854-63. 
4. Donovan B, Franklin N, Guy R, Grulich AE, Regan DG, Ali $\mathrm{H}$, et al. Quadrivalent human papillomavirus vaccination and trends in genital warts in Australia: analysis of national sentinel surveillance data. Lancet Infect Dis. 2011;11(1):39-44.

5. Fairley CK, Hocking JS, Gurrin LC, Chen MY, Donovan B, Bradshaw CS. Rapid decline in presentations of genital warts after the implementation of a national quadrivalent human papillomavirus vaccination programme for young women. Sex Transm Infect. 2009;85(7):499-502.

6. Munoz N, Kjaer SK, Sigurdsson K, Iversen OE, Hernandez-Avila M, Wheeler $\mathrm{CM}$, et al. Impact of human papillomavirus (HPV)-6/11/16/18 vaccine on all HPVassociated genital diseases in young women. J Natl Cancer Inst. 2010;102(5):325-39.

7. Tota J, Mahmud SM, Ferenczy A, Coutlée F, Franco EL. Promising strategies for cervical cancer screening in the post-human papillomavirus vaccination era. Sex Health. 2010;7(3):376-82.

8. Kliewer EV, Demers AA, Brisson M, Severini A, Lotocki R, Elias B, et al. The Manitoba human papillomavirus vaccine surveillance and evaluation system. Health Rep. 2010;21(2):37-42.

9. Bouvard V, Baan R, Straif K, Grosse Y, Secretan B, El Ghissassi F, et al. A review of human carcinogens-Part B: biological agents. Lancet Oncol. 2009;10(4):321-2.

10. Higuchi R. Simple and rapid preparation of samples for PCR. In: Erlich HA, editor. PCR Technology. Principles and Applications for DNA Amplification. New York: Stockton Press; 1989. p. 31-8.

11. Josefsson A, Magnusson P, Gyllensten U. Human papillomavirus detection by PCR and typing by Dot-Blot. In: Peeling RW, Sparling PF, editors. Sexually Transmitted Diseases. Methods and Protocols. Totowa (NJ): Humana Press; 1999. p. 171-93.

12. Gravitt PE, Peyton CL, Alessi TQ, Wheeler CM, Coutlée F, Hildesheim A, et al. Improved amplification of genital human papillomaviruses. J Clin Microbiol. 2000;38(1):357-61.
13. de Roda Husman AM, Walboomers JM, van den Brule AJ, Meijer CJ, Snijders PJ. The use of general primers GP5 and GP6 elongated at their 3' ends with adjacent highly conserved sequences improves human papillomavirus detection by PCR. J. Gen. Virol. 1995 Apr;76 (Pt 4):1057-62.

14. Evander M, Edlund K, Bodén E, Gustafsson A, Jonsson M, Karlsson R, et al. Comparison of a one-step and a two-step polymerase chain reaction with degenerate general primers in a population-based study of human papillomavirus infection in young Swedish women. J Clin Microbiol. 1992;30(4):987-92.

15. Strauss S, Jordens JZ, Desselberger U, Gray JJ. Single-tube real-time nested polymerase chain reaction for detecting human papillomavirus DNA. Diagn Mol Pathol. 2000;9(3):151-7.

16. Bulkmans NW, Berkhof J, Rozendaal L, van Kemenade FJ, Boeke AJ, Bulk S, et al. Human papillomavirus DNA testing for the detection of cervical intraepithelial neoplasia grade 3 and cancer: 5 -year follow-up of a randomised controlled implementation trial. Lancet. 2007;370(9601):1764-72.

17. Zubach V, Smart G, Ratnam S, Severini A. Novel microsphere-based method for detection and typing of 46 mucosal human papillomavirus types. J Clin Microbiol. 2012;50(2):460-4.

18. Rebbapragada A, Perusini A, Di Prima A, Salit I, Goleski V, Severini A. Evaluation of test performance characteristics and operational parameters of $5 \mathrm{HPV}$ genotyping assays. 26th International Papillomavirus Conference, Montreal, Canada, July 3-8: 2010.

19. de Villiers EM, Fauquet C, Broker TR, Bernard HU, Zur Hausen $\mathrm{H}$. Classification of papillomaviruses. Virology. 2004;324(1):17-27.

20. de Sanjosé S, Diaz M, Castellsagué X, Clifford G, Bruni L, Munoz N, et al. Worldwide prevalence and genotype distribution of cervical human papillomavirus DNA in women with normal cytology: a meta-analysis. Lancet Infect Dis. 2007;7 (7):453-9.
21. Dunne EF, Unger ER, Sternberg $M$, McQuillan G, Swan DC, Patel SS, et al. Prevalence of HPV infection among females in the United States. JAMA. 2007;297(8):813-9.

22. Bruni L, Diaz M, Castellsagué X, Ferrer E, Bosch FX, de Sanjosé S. Cervical human papillomavirus prevalence in 5 continents: meta-analysis of 1 million women with normal cytological findings. J Infect Dis. 2010;202(12):1789-99.

23. Clifford GM, Rana RK, Franceschi S, Smith JS, Gough G, Pimenta JM. Human papillomavirus genotype distribution in low-grade cervical lesions: comparison by geographic region and with cervical cancer. Cancer Epidemiol Biomarkers Prev. 2005;14(5):1157-64.

24. Smith JS, Lindsay L, Hoots B, Keys J, Franceschi S, Winer R, et al. Human papillomavirus type distribution in invasive cervical cancer and high-grade cervical lesions: a meta-analysis update. Int J Cancer. 2007;121(3):621-32.

25. Arbyn M, Sasieni P, Meijer CJ, Clavel C, Koliopoulos G, Dillner J. Chapter 9: clinical applications of HPV testing: a summary of meta-analyses. Vaccine. 2006;24 Suppl 3:S3/78-89.

26. Naucler P, Ryd W, Tornberg S, Strand A, Wadell G, Hansson BG, et al. HPV type-specific risks of high-grade CIN during 4 years of follow-up: a population-based prospective study. $\mathrm{Br} \mathrm{J}$ Cancer. 2007;97(1):129-32.

27. Burchell AN, Winer RL, de Sanjosé S, Franco EL. Chapter 6: epidemiology and transmission dynamics of genital HPV infection. Vaccine. 2006;24(Supplement 3):S52-S61.

28. Moore RA, Ogilvie G, Fornika D, Moravan V, Brisson M, Amirabbasi-Beik M, et al. Prevalence and type distribution of human papillomavirus in 5,000 British Columbia women-implications for vaccination. Cancer Causes Control. 2009;20(8):1387-96.

29. Sellors JW, Mahony JB, Kaczorowski J, Lytwyn A, Bangura $\mathrm{H}$, Chong S, et al. Prevalence and predictors of human papillomavirus infection in women in Ontario, Canada. Survey of HPV in Ontario Women (SHOW) Group. CMAJ. 2000;163(5):503-8. 
30. Kurz J, Mitra K, Adam R, Miao X, MacKay JS, Isa NN, et al. PCR detection and typing of genital papillomavirus in a New Brunswick population. Int J Cancer. 1993;55(4):604-8.

31. Young TK, McNicol P, Beauvais J. Factors associated with human papillomavirus infection detected by polymerase chain reaction among urban Canadian aboriginal and non-aboriginal women. Sex Transm Dis. 1997;24(5):293-8.

32. Trottier H, Franco EL. The epidemiology of genital human papillomavirus infection. Vaccine. 2006;24(Suppl 1):S1-15.

33. Scheurer ME, Tortolero-Luna G, Adler-Storthz K. Human papillomavirus infection: biology, epidemiology, and prevention. Int $\mathrm{J}$ Gynecol Cancer. 2005;15(5):727-46.

34. Moscicki AB, Schiffman M, Kjaer S, Villa LL. Chapter 5: updating the natural history of HPV and anogenital cancer. Vaccine. 2006;24(Supplement 3):S42-S51.

35. Trottier H, Burchell AN. Epidemiology of mucosal human papillomavirus infection and associated diseases. Public Health Genomics. 2009;12(5-6):291-307.

36. Winer RL, Lee SK, Hughes JP, Adam DE, Kiviat NB, Koutsky LA. Genital human papillomavirus infection: incidence and risk factors in a cohort of female university students. Am J Epidemiol. 2003;157(3):218-26.

37. Ho GYF, Bierman R, Beardsley L, Chang CJ, Burk RD. Natural history of cervicovaginal papillomavirus infection in young women. N Engl J Med. 1998;338(7):423-8.

38. Moscicki AB, Hills N, Shiboski S, Powell K, Jay N, Hanson E, et al. Risks for incident human papillomavirus infection and low-grade squamous intraepithelial lesion development in young females. JAMA. 2001;285(23):2995-3002.

39. Goodman MT, Shvetsov YB, McDuffie K, Wilkens LR, Zhu X, Thompson PJ, et al. Prevalence, acquisition, and clearance of cervical human papillomavirus infection among women with normal cytology: Hawaii Human Papillomavirus Cohort Study. Cancer Res. 2008;68(21):8813-24.
40. Giuliano AR, Harris R, Sedjo RL, Baldwin S, Roe D, Papenfuss MR, et al. Incidence, prevalence, and clearance of type-specific human papillomavirus infections: The Young Women's Health Study. J Infect Dis. 2002;186(4):462-9.

41. Sellors JW, Karwalajtys TL, Kaczorowski J, Mahony JB, Lytwyn A, Chong S, et al. Incidence, clearance and predictors of human papillomavirus infection in women. CMAJ. 2003;168(4):421-5.

42. Decker K, Demers A, Chateau D, Musto G, Nugent Z, Lotocki R, et al. Papanicolaou test utilization and frequency of screening opportunities among women diagnosed with cervical cancer. Open Med. 2009;3(3):140-7.

43. Boulanger JC, Fauvet R, Urrutiaguer S, Drean Y, Sevestre H, Ganry O, et al. [Cytological history of cases of invasive cervical cancer diagnosed in France in 2006.] Gynecol Obstet Fertil. 2007;35(9):764-71.

44. van der Aa MA, Schutter EM, LooijenSalamon M, Martens JE, Siesling S. Differences in screening history, tumour characteristics and survival between women with screen-detected versus not screendetected cervical cancer in the east of The Netherlands, 1992-2001. Eur J Obstet Gynecol Reprod Biol. 2008;139(2):204-9.

45. Priest P, Sadler L, Peters J, Crengle S, Bethwaite P, Medley G, et al. Pathways to diagnosis of cervical cancer: screening history, delay in follow up, and smear reading. BJOG. 2007;114(4):398-407.

46. Depuydt CE, Arbyn M, Benoy IH, Vandepitte J, Vereecken AJ, Bogers JJ. Quality control for normal liquid-based cytology: rescreening, high-risk HPV targeted reviewing and/or high-risk HPV detection? J Cell Mol Med. 2009;13(9B):4051-60. 